\title{
Article \\ Conduction Disorders during Sinus Rhythm in Relation to Atrial Fibrillation Persistence
}

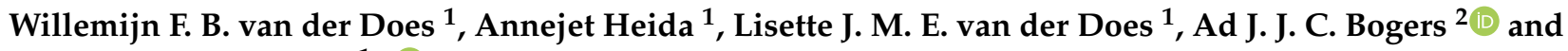 \\ Natasja M. S. de Groot 1,*(D) \\ 1 Department of Cardiology, Erasmus Medical Center, 3015 CN Rotterdam, The Netherlands; \\ w.vanderdoes@erasmusmc.nl (W.F.B.v.d.D.); a.heida@erasmusmc.nl (A.H.); \\ j.vanderdoes@erasmusmc.nl (L.J.M.E.v.d.D.) \\ 2 Department of Cardiothoracic Surgery, Erasmus Medical Center, 3015 CN Rotterdam, The Netherlands; \\ a.j.j.c.bogers@erasmusmc.nl \\ * Correspondence: n.m.s.degroot@erasmusmc.nl; Tel.: +31-10-703-4479; Fax: + 31-10-703-5258
}

check for updates

Citation: van der Does, W.F.B.; Heida, A.; van der Does, L.J.M.E.; Bogers, A.J.J.C.; de Groot, N.M.S. Conduction Disorders during Sinus Rhythm in Relation to Atrial Fibrillation Persistence. J. Clin. Med. 2021, 10, 2846. https://doi.org/ $10.3390 / \mathrm{jcm} 10132846$

Academic Editors: Gregory Y. H. Lip and Inna P. Gladysheva

\section{Received: 21 April 2021}

Accepted: 24 June 2021

Published: 27 June 2021

Publisher's Note: MDPI stays neutral with regard to jurisdictional claims in published maps and institutional affiliations.

Copyright: (c) 2021 by the authors. Licensee MDPI, Basel, Switzerland. This article is an open access article distributed under the terms and conditions of the Creative Commons Attribution (CC BY) license (https:// creativecommons.org/licenses/by/ $4.0 /)$.

\begin{abstract}
Classification of atrial fibrillation (AF) is currently based on clinical characteristics. However, classifying AF using an objective electrophysiological parameter would be more desirable. The aim of this study was to quantify parameters of atrial conduction during sinus rhythm (SR) using an intra-operative high-resolution epicardial mapping approach and to relate these parameters to clinical classifications of AF. Patients were divided according to the standard clinical classification and spontaneous termination of AF episodes. The HATCH score, a score predictive of AF progression, was calculated, and surface ECGs were evaluated for signs of interatrial block. Conduction disorders mainly differed at Bachmann's bundle (BB). Activation time (AT) at BB was longer in persistent AF patients (AT-BB: 75 (53-92) ms vs. $55(40-76) \mathrm{ms}, p=0.017)$, patients without spontaneous termination of AF episodes (AT-BB: 53.5 (39.6-75.8) ms vs. 72.0 (49.6-80.8) ms, $p=0.009$ ) and in patients with a P-wave duration $\geq 120 \mathrm{~ms}(64.3$ (52.3-93.0) ms vs. 50.5 (39.6-56.6) ms, $p=0.014)$. HATCH scores also correlated positively to AT-BB (rho 0.326, $p=0.029$ ). However, discriminatory values of electrophysiological parameters, as calculated using ROC-curves, were limited. These results may reflect shortcomings of clinical classifications and further research is needed to establish an objective substrate-based classification of AF.
\end{abstract}

Keywords: atrial fibrillation; conduction disturbances; high-resolution mapping; AF persistence

\section{Introduction}

Atrial fibrillation (AF) is classified in the ESC guidelines according to the clinical presentation and duration of AF episodes. Pulmonary vein isolation is recommended for paroxysmal AF patients with symptomatic recurrences of AF on antiarrhythmic drug therapy; however, late recurrences of AF after ablation are common [1,2]. The unpredictable, moderate success rate of pulmonary vein isolation is not only caused by reconduction but is also likely the result of differences in severity and extent of electropathology in patients with the same AF subtype [3-5]. In the latter case, a different classification based on AF-related electropathology should be considered to select patients who will benefit from pulmonary vein isolation.

Previously, mapping studies have shown differences in conduction characteristics between paroxysmal and persistent AF patients. During pacing, conduction slowing was more pronounced in the left atrium (LA) and Bachmann's bundle (BB) in patients with persistent AF, compared to patients with paroxysmal AF [6,7]. During sinus rhythm (SR), a longer activation time of the LA was found in persistent AF patients compared to paroxysmal AF patients [8-10]. In contrast, in the pulmonary vein area (PVA) no relation was found between severity of conduction disorders in SR and AF subtypes [11]. 
These contradictory results raise the question whether differences between paroxysmal and persistent AF are confined to specific anatomical regions. It is unknown whether there are discriminatory differences in characteristics of conduction disorders during SR in both atria between paroxysmal and persistent AF patients. If differences are present, an objective classification based on electropathology could be established.

The goal of this study is, therefore, to compare conduction disorders during SR between patients with paroxysmal and persistent AF using a high resolution epicardial mapping approach covering the right atrium (RA), BB, LA and the PVA. As the standard classification of AF might be limited in its discriminative ability, we also compare conduction disorders between patients with or without spontaneous termination of AF episodes and between patients with or without ECG characteristics identifying interatrial block. We also correlate conduction characteristics to the HATCH score, a score predicting progression of paroxysmal AF [12].

\section{Experimental Section}

\subsection{Study Population}

Patients ( $\geq 18$ years) undergoing elective cardiac surgery for either coronary bypass grafting, aortic or mitral valve surgery or a combination of these procedures were included. This study was approved by the Medical Ethical Committee in the Erasmus Medical Center (MEC 2010-054 and MEC 2014-393) and follows the declaration of Helsinki principles. Written informed consent was obtained from all patients. Clinical data was collected from electronic records; patients with a history of AF were selected for this study. In line with the ESC guidelines, patients with documentation (ECG, ECG description) of AF episodes up to 7 days, or with AF episodes cardioverted within 7 days were classified as paroxysmal AF. Patients with documentation of AF episodes longer than 7 days or longer than a year were classified as persistent and long-standing persistent $\mathrm{AF}$, respectively. In patients with paroxysmal AF, the HATCH score (hypertension, age ( $>75$ years), transient ischemic attack or stroke, chronic obstructive pulmonary disease and heart failure) was calculated. Additionally, patients were subdivided in 2 groups according to whether AF episodes pre-operatively terminated spontaneously or required intervention (electrical or chemical cardioversion). In patients presenting with SR before surgery, ECGs were evaluated for signs of advanced interatrial block (a-IAB) and the duration of the P-wave as described by Bayes de Luna et al. [13]

\subsection{Mapping Procedure}

Epicardial mapping was performed during open heart surgery, prior to initiation of extracorporal circulation. A steel wire was fixed into subcutaneous tissue serving as an indifferent electrode. A bipolar epicardial pacemaker wire, stitched into the right atrium free wall, served as a reference electrode. Mapping was performed during SR with either a custom-made 128-unipolar electrode array or a 192-unipolar electrode array, inter-electrode distances of both arrays were $2 \mathrm{~mm}$.

Mapping was conducted by shifting the electrode array consecutively over the right atrium (RA) to BB and the LA including PVA, as previously described in detail [14]. This mapping method, including positioning of the reference electrode, has been standardized and used for more than a decade by a group of dedicated cardiothoracic surgeons who perform these mapping procedures. If the rhythm at the onset of the mapping procedure was AF, electrical cardioversion was performed to restore SR. At every site, five seconds of SR were recorded, including a surface ECG, bipolar reference and a calibration signal of $2 \mathrm{mV}$ and $1000 \mathrm{~ms}$. Recordings were amplified with gain 1000, sampled with a rate of $1 \mathrm{kHz}$, filtered with bandwidth $0.5-400 \mathrm{~Hz}$, analogue-to-digital converted (16 bits) and stored on hard disk. 


\subsection{Data Processing}

Using dedicated mapping software, unipolar electrograms were analyzed. As unipolar electrograms provide more detailed information, especially in our high-resolution mapping setting, we chose to use unipolar instead of bipolar electrograms. The steepest part of the negative deflection was automatically annotated if the amplitude exceeded the noise level in the channel with a probability of $99.95 \%$. Local activation times (LAT) of these annotations were translated into an activation map for every sinus beat. All markings were manually verified. Electrogram files were excluded when deflections were marked in less than $40 \%$ of the mapping area due to recordings of poor quality (low signal to noise ratios).

For each electrode the conduction time (CT) was calculated as the difference in LAT with the bordering right and lower electrodes, as illustrated in Figure 1. As in prior mapping studies, conduction delay (CD) was defined as CTs $>6$ and $<12 \mathrm{~ms}$, conduction block (CB) was defined as CTs $>11 \mathrm{~ms}$. These cutoffs for $\mathrm{CB}$ were determined, as explained in previous work, based on the slowest conduction during longitudinal propagation [15]. When $\mathrm{CB}$ lines connected with $\mathrm{CD}$ lines, this was labeled as a continuous conduction delay and block ( $\mathrm{CCDCB}$ ) line. These parameters are shown in detail in the upper right panel in Figure 1. All conduction parameters were calculated per mapping site (e.g., LA1, LA2) and then translated to an average, maximum or sum for the corresponding location (e.g., LA). Total activation time (TAT) and activation time (AT) for every location were determined by comparing the first and last activation of the corresponding mapping sites in relation to the bipolar reference electrode. At BB, as fiber direction in this location is known, we evaluated the direction of $C B$ lines as being mostly perpendicular to, in parallel with, or diagonal on the fiber direction. If a perpendicular $\mathrm{CB}$ line completely isolated the right and left side of $\mathrm{BB}$, this was scored as an isolating $\mathrm{CB}$ line.
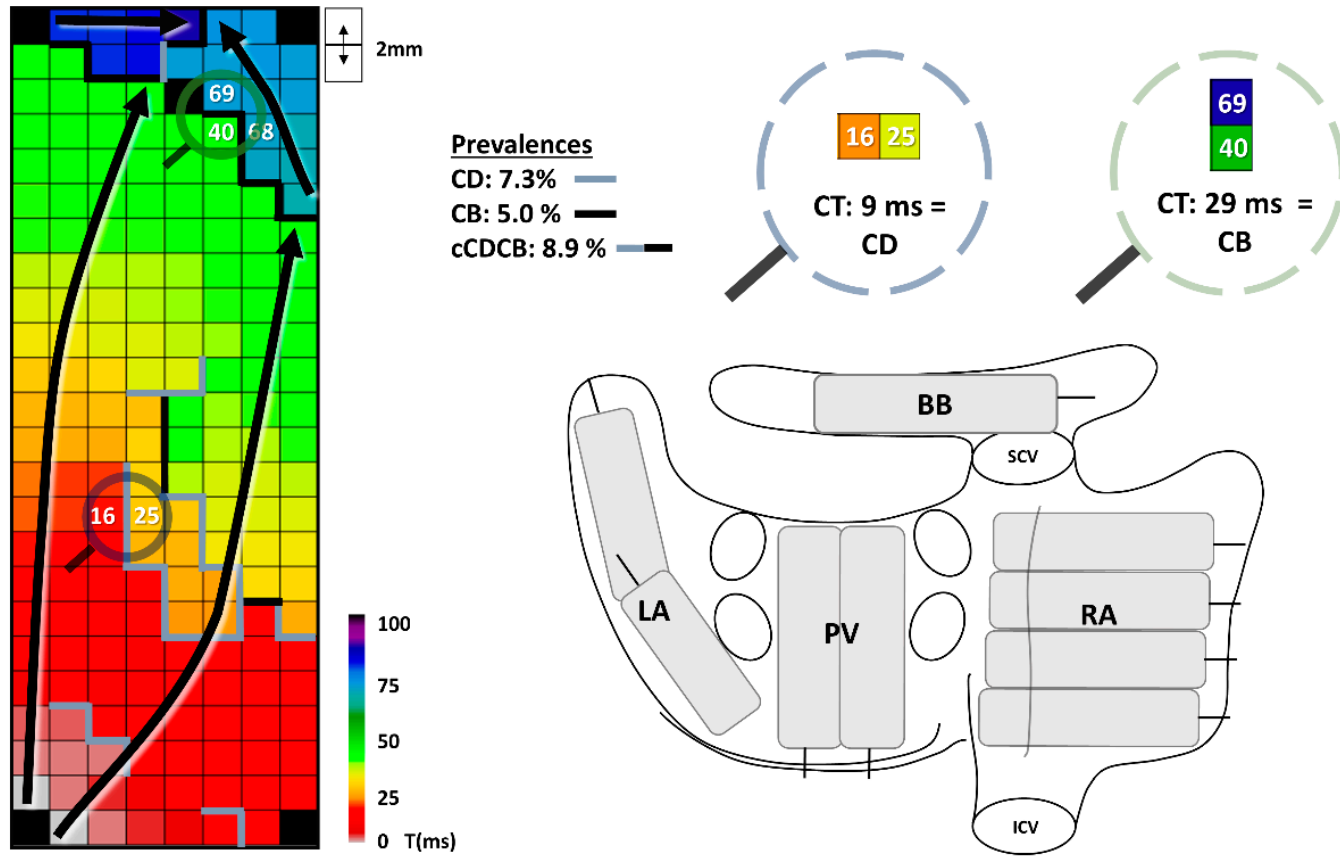

Figure 1. Conduction delay and block. The left panel shows a color-coded activation map of BB with conduction disturbances and arrows showing the direction of wavefront propagation. The upper right panel shows the corresponding prevalences of conduction delay (CD), conduction block (CB) and continuous conduction delay and block (cCDCB). CT: conduction time; the time difference between two electrodes. The lower right panel shows an overview of the mapping locations on a posterior view of the heart. BB: Bachmann's bundle; ICV: internal vena cava; LA: left atrium; PVA: pulmonary vein area; SCV: superior vena cava; RA: right atrium. 


\subsection{Statistical Analysis}

Normally distributed data are described by mean \pm standard deviation, skewed data by median (interquartile range) and categorical data by absolute number (percentage). Differences between groups were calculated with a student's $t$-test or Mann-Whitney U. When data was categorical, chi-square testing or an exact test was performed if appropriate. Correlations between $\mathrm{HATCH}$ score and electrophysiological parameters were evaluated using Spearman rank correlation. Multiple linear regression was performed to correct for confounding in continuous data, using a log transformation with the parameters AT-BB and AT-LA as the criteria for linear regression were violated. We constructed ROC-curves and evaluated the area under the curve (AUC) to determine the discriminatory value of various electrophysiological parameters. A $p$-value $<0.05$ was considered statistically significant. All statistical analyses were performed with IBM SPSS statistics for Windows, version 25 (IBM Corp., Armonk, NY, USA).

\section{Results}

\subsection{Patient Characteristics}

The study population consisted of 71 patients (age $72 \pm 7$ years, 28 (39\%) female); baseline characteristics are shown in Table 1 with patients divided according to clinical classification. Patients had either paroxysmal $(n=47,66 \%)$ or persistent $(n=24,34 \%)$ AF, including three patients with long-standing persistent AF. Indications for cardiac surgery were coronary artery bypass grafting $(n=20,28 \%)$, aortic or mitral valve surgery or a combination of valvular and bypass surgery $(n=51,72 \%)$. There were no significant differences in baseline characteristics between patients with paroxysmal and persistent AF. Cardioversion before mapping was necessary in three $(6.4 \%)$ patients from the paroxysmal AF group, and 17 (70.8\%) patients from the persistent AF group.

Table 1. Baseline characteristics.

\begin{tabular}{cccc}
\hline & $\begin{array}{c}\text { Paroxysmal AF } \\
(\boldsymbol{n}=\mathbf{4 7})\end{array}$ & $\begin{array}{c}\text { Persistent AF } \\
(\boldsymbol{n}=\mathbf{2 4})\end{array}$ & $\boldsymbol{p}$ \\
\hline Age (years) & $72.2 \pm 7.3$ & $71.0 \pm 6.8$ & 0.496 \\
\hline Gender: female & $21(44.7)$ & $7(29.2)$ & 0.206 \\
\hline BMI $\left(\mathrm{kg} / \mathrm{m}^{2}\right)$ & $27.3 \pm 4.4$ & $28.7 \pm 4.3$ & 0.198 \\
\hline Years since diagnosed AF & $1.0[0.3-5.0]$ & $1.0[0.4-5.5]$ & 0.823 \\
\hline Diabetes mellitus & $12(25.5)$ & $5(20.8)$ & 0.661 \\
\hline Hypertension & $29(61.7)$ & $15(62.5)$ & 0.948 \\
\hline Dyslipidemia & $13(27.7)$ & $6(25.0)$ & 0.811 \\
\hline History of myocardial infarction & $10(21.3)$ & $3(12.5)$ & 0.521 \\
\hline AADs: & $1(2.1) \dagger$ & & \\
Class I & $27(57.4)$ & $14(58.3)$ & 0.845 \\
Class II & $12(25.5)$ & $6(25.0)$ & \\
Class III & 0 & $2(8.3) \dagger$ & \\
Class IV & & & \\
Surgical indication: & $32(68.1)$ & $19(79.2)$ & \\
(i) VHD & 12 & 6 & \\
AVD & 5 & 2 & \\
AVD + CABG & 10 & 10 & \\
MVD & $15(31.9)$ & $5(20.8)$ & \\
MVD + CABG & & & \\
CABG & $5326 \ddagger$ & \\
\hline
\end{tabular}


Table 1. Cont.

\begin{tabular}{cccc}
\hline & $\begin{array}{c}\text { Paroxysmal AF } \\
(\boldsymbol{n}=\mathbf{4 7})\end{array}$ & $\begin{array}{c}\text { Persistent AF } \\
(\boldsymbol{n}=\mathbf{2 4})\end{array}$ & $\boldsymbol{p}$ \\
\hline Left ventricular EF: & & & \\
Normal $(\geq 50 \%)$ & $39(83.0)$ & $14(58.3)$ & 0.101 \\
Mild impairment $(40-49 \%)$ & $5(10.6)$ & $6(25.0)$ & \\
Moderate impairment $(30-39 \%)$ & $2(4.3)$ & $4(16.7)$ & 0 \\
Severe impairment $(\leq 30 \%)$ & $1(2.1)$ & $21 / 24(87.5 \%)$ II & 0.207 \\
\hline LA enlargement $\S$ & $32 / 43(74.4 \%) \mathbb{I}$ & 0.568 \\
\hline LAVI $\left(\mathrm{mL} / \mathrm{m}^{2}\right)$ & $41(33-56)$ & $44(38-50)$ & $(n=18) \mathbb{I}$ \\
\hline
\end{tabular}

AAD: antiarrhythmic drugs; AVD: aortic valve disease; BMI: body mass index; CABG: coronary artery bypass graft; EF: ejection fraction; LAVI: left atrial volume index; MVD: mitral valve disease; (i)VHD: (ischemic) valvular heart disease; $†$ these patients also used another class of AADs; $\ddagger$ comparison between (i)VHD and CABG; $\S$ left atrial enlargement was defined as LAVI $>28 \mathrm{~mL} / \mathrm{m}^{2}$; II indicates missing data.

The duration of the longest AF episode in patients with paroxysmal AF was less than 1 day for 18 (38.2\%) patients and between $1-6$ days for 10 patients $(21.3 \%)$. For the remaining patients classified as paroxysmal $\mathrm{AF}(n=19,40.4 \%)$ there was no information on duration of AF episodes. In the persistent AF group, the longest AF episode was 3 (1-5.5) months, ranging between 2 weeks and 2 years. SR cycle length was 874.1 (760.8-1012.8) $\mathrm{ms}$ in the paroxysmal AF group and 857.7 (751.4-1005.6) $\mathrm{ms}$ in the persistent AF group $(p=0.498)$.

\subsection{Conduction Disorders in Paroxysmal and Persistent AF Patients}

The prevalence of $\mathrm{CB}$ in the entire atria in the paroxysmal $\mathrm{AF}$ group ranged between $0.5 \%$ and $8.7 \%$ and between $0.4 \%$ and $7.0 \%$ in the persistent AF group. As demonstrated in Supplemental Table S1, these large ranges were present at all locations. For all locations, there were no differences in prevalences of $\mathrm{CD}, \mathrm{CB}$ and $\mathrm{CCDCB}$ between the paroxysmal and persistent $\mathrm{AF}$ patients. A higher number of $\mathrm{CCDCB}$ lines was found at $\mathrm{BB}$ in the persistent AF group (2 (1-3) vs. $3(2-4), p=0.040)$. However, there were no differences in length of (longest) lines of $\mathrm{CD}, \mathrm{CB}$, or $\mathrm{CCDCB}$ between the paroxysmal and persistent $\mathrm{AF}$ group in any location.

Conduction disorders particularly occurred at BB. Perpendicular orientation of the longest $\mathrm{CB}$ line at $\mathrm{BB}$ was more frequently present in patients with persistent $\mathrm{AF}(n=19$, $82.6 \%)$, than in patients with paroxysmal AF $(n=22,52.4 \%), p=0.028$. There was no difference in prevalence of isolating $C B$ lines (upper panel Figure 2). The exact number of perpendicularly orientated $\mathrm{CB}$ line parts was evaluated as explained in the left part of the middle panel of Figure 2. Patients with persistent AF had more perpendicularly orientated parts of CB line than paroxysmal AF patients (middle panel Figure 2; PAF: $13.0 \pm 7.7$, persAF: $18.3 \pm 10.0, p=0.045)$. The number of perpendicularly orientated parts of $\mathrm{CB}$ was correlated with AT of BB (lower panel Figure 4; rho 0.605, $p<0.0005$ ).

As demonstrated in Figure 3, TAT in the persistent AF group (156 (135-172) ms) was longer than TAT in the paroxysmal AF group (137 (122-154) ms), $p=0.034$. This distinction was caused by a longer AT-LA (45 (29-55) ms vs. $30(25-39) \mathrm{ms}, p=0.010)$ and AT-BB (75 (53-92) ms vs. 55 (40-76) ms, $p=0.017)$ in the persistent AF group.

To exclude a confounding effect of clinical parameters, we performed a multivariate analysis for TAT, AT-BB and AT-LA (Supplemental Table S2). Persistent AF remained an independent predictor of a longer TAT $(p=0.031)$ and AT-BB $(p=0.008)$, but not of a longer AT-LA $(p=0.286)$. However, the multivariate analyses did not include all patients, as LAVI was not available for every patient. The multivariate analyses showed female gender and age as independent predictors for a longer AT-BB. 


\begin{tabular}{|c|c|c|c|}
\hline $\begin{array}{c}\text { Isolating } \\
\text { perpendicular CB } \\
\text { line }\end{array}$ & Yes & No & $p$ value \\
\hline PAF & $48.9 \%(n=22)$ & $51.1 \%(n=23)$ & \\
\hline persAF & $54.2 \%(n=13)$ & $45.8 \%(n=11)$ & 0.676 \\
\hline
\end{tabular}

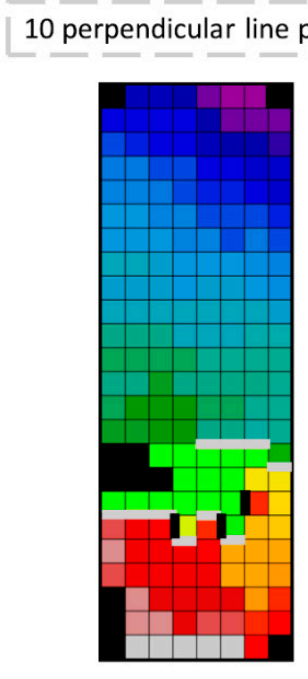

$\left[\begin{array}{r}\text { parallel line part } \\ -\end{array}\right.$
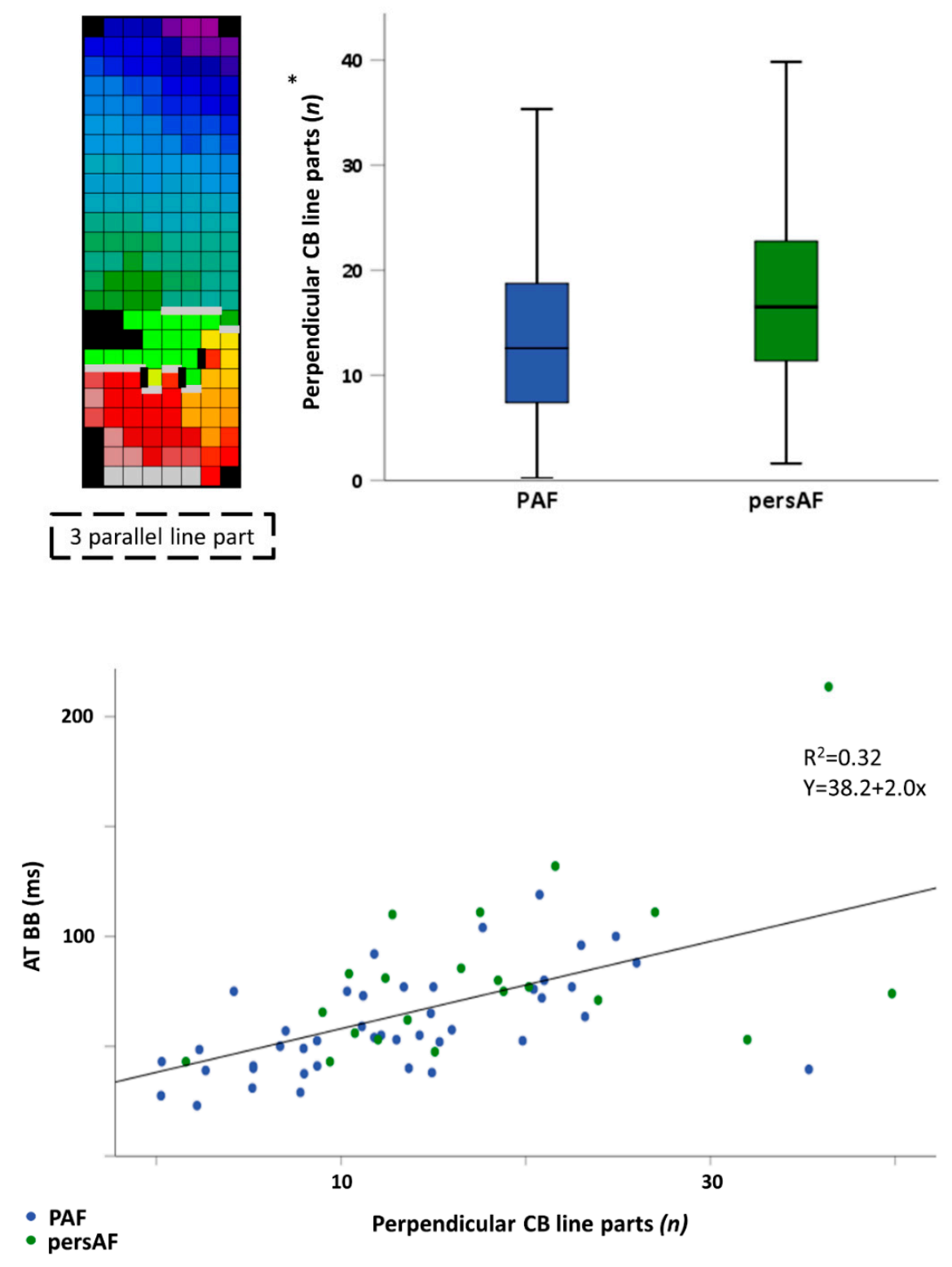

Figure 2. Paroxysmal vs. persistent AF patients: conduction disorders at Bachmann's Bundle. The table in the upper panel shows percentages of PAF and persAF patients with an isolating perpendicularly orientated CB line. The left part of the middle panel illustrates how the number of parallel and perpendicular orientated CB line parts was determined. An activation map is depicted with gray perpendicular CB line parts and black parallel CB line parts. The right part of the middle panel shows the difference in perpendicular CB line parts between PAF and persAF patients. The scatterplot in the lower panel depicts the relation between amount of perpendicular $\mathrm{CB}$ line parts and AT at BB in all patients. BB: Bachmann's bundle; CB: conduction block; PAF: paroxysmal AF; persAF: persistent AF; TAT: total activation time; ${ }^{*} p=0.045$. 


\section{Total activation time (ms)}

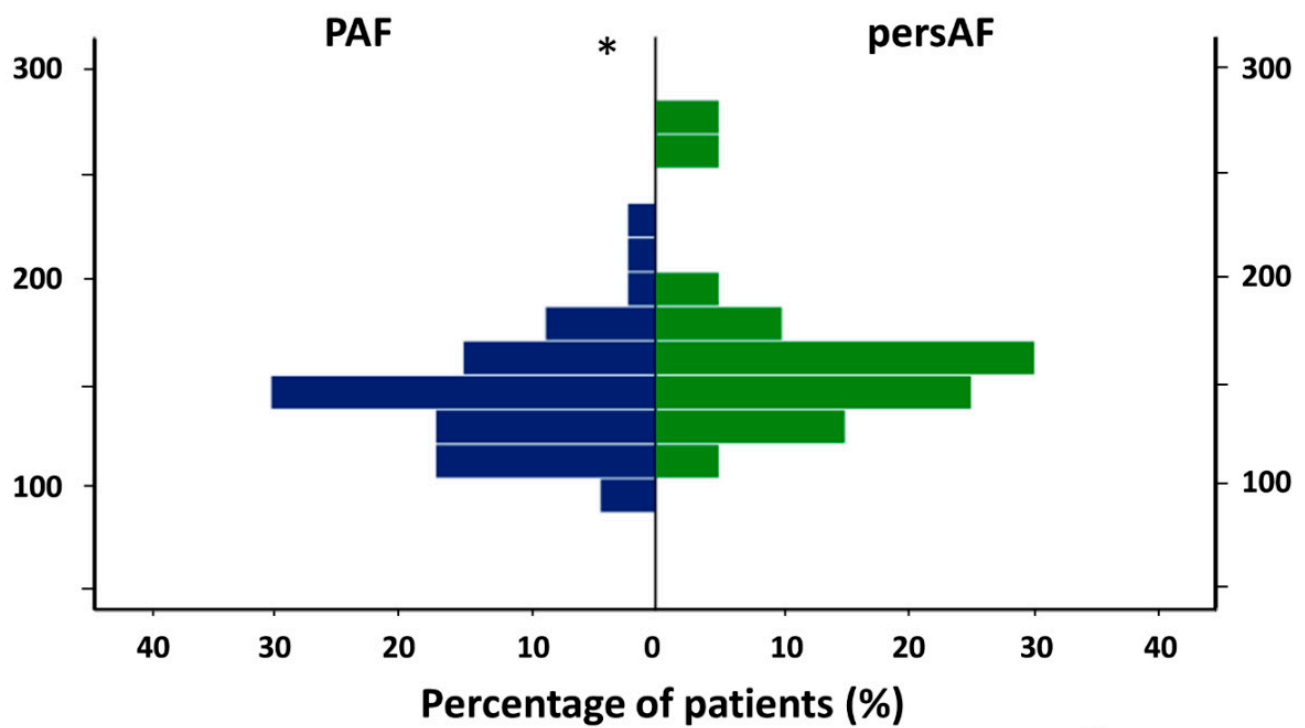

RA
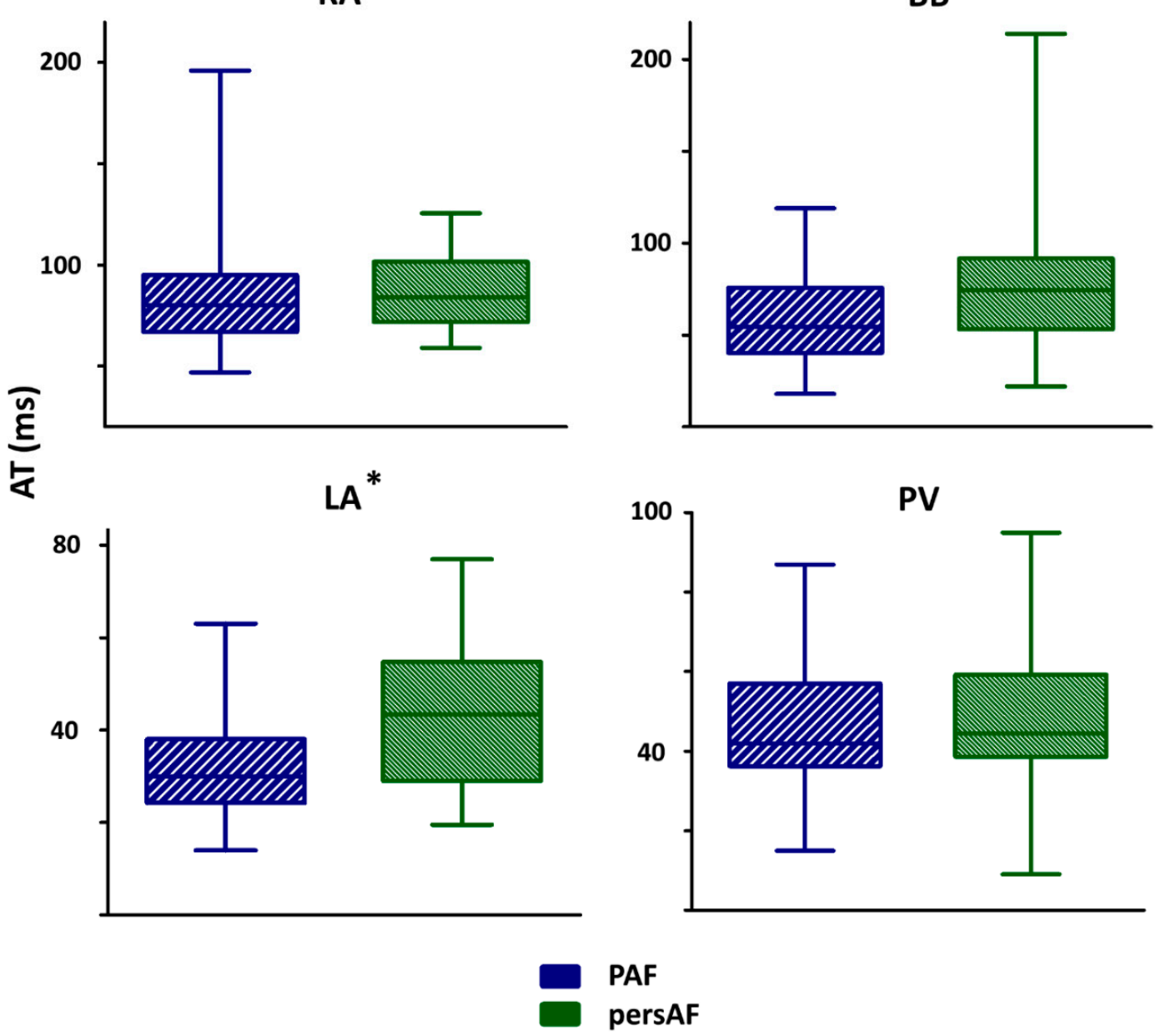

Figure 3. Paroxysmal vs. persistent AF patients: activation times. The upper panel shows the distribution of total activation time for the PAF group and persAF group. The lower panel depicts ATs for each location separately in the PAF and persAF group. BB: Bachmann's bundle; LA: left atrium; PVA: pulmonary vein area; PA: paroxysmal AF; persAF: persistent AF; RA: right atrium; AT: activation time; ${ }^{*}$ total activation time; $p=0.034$, BB: $p=0.017$, LA: $p=0.010$. 
An example of the distribution of the prevalence of $\mathrm{CB}$ and AT in the atria of a typical paroxysmal and typical persistent AF patient is demonstrated in Figure 4. The paroxysmal AF patient was diagnosed 3 months before surgery and the persistent AF patient was diagnosed 10 months before surgery. Both underwent mitral valve surgery. In the upper panel of Figure 4, comparable prevalences of $\mathrm{CB}$ are seen in the paroxysmal and persistent AF patient in all areas except $B B$. In the patient with persistent $A F$, an isolating $C B$ line in $\mathrm{BB}$ is seen; a thick black $\mathrm{CB}$ line which connects the lower and upper side of the mapping array. Most CB lines in the RA in this figure, especially apparent in the paroxysmal AF patient, are found in the crista terminalis area. In the lower panel of Figure 4, the AT for every location is depicted. The AT of LA, PVA and BB are longer in the persistent AF patient, while the AT of RA is longer in the paroxysmal AF patient.

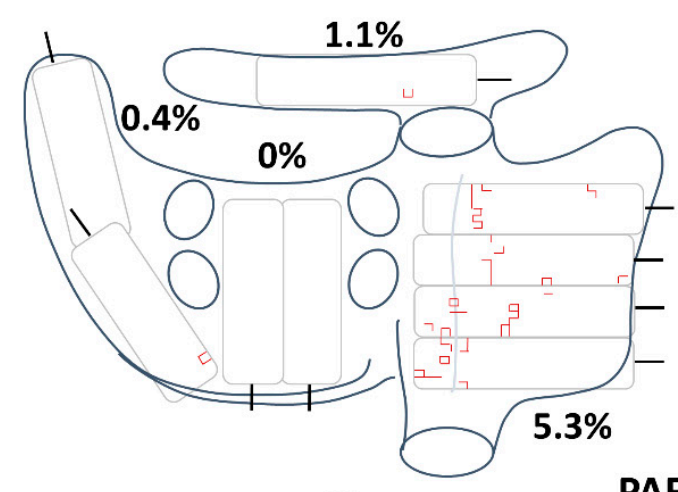

PAF // persAF

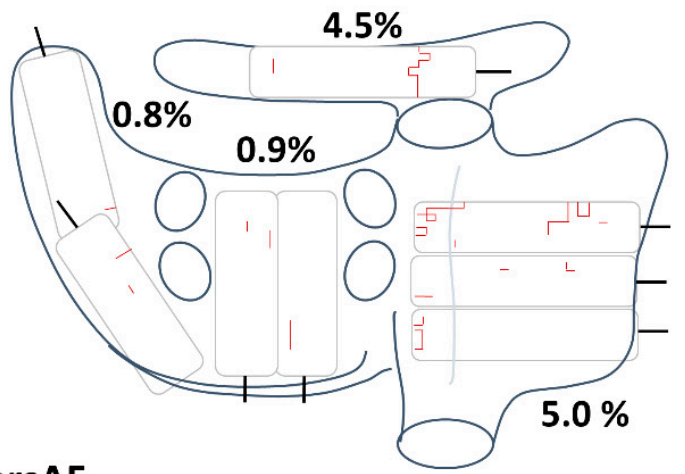

$39 \mathrm{~ms}$

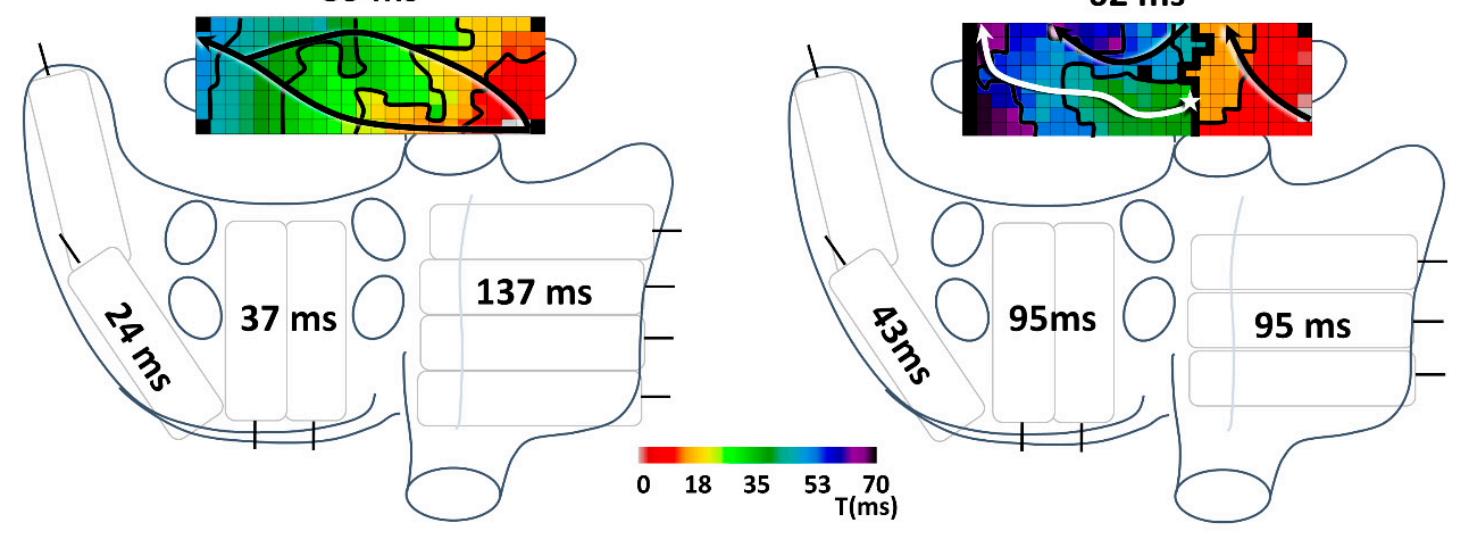

$62 \mathrm{~ms}$

Figure 4. Typical paroxysmal and persistent AF patient. The distribution of CB prevalence (upper panel) and AT (lower panel) is seen in a PAF (left) and a persAF patient (right). In the lower panel, two activation maps are demonstrated at BB with $10 \mathrm{~ms}$ isochrones, arrows showing the direction of wavefront propagation and thick black lines indicating CB area. The paroxysmal AF patient, 69 years old, was diagnosed 3 months before he underwent mitral valve surgery. He had been experiencing palpitations for minutes to hours at a time and AF occurred during a cardiac exercise stress test while he experienced similar palpitations. The persistent AF patient, 64 years old, was diagnosed with AF 10 months before he underwent mitral valve surgery. As duration of AF was unknown at the time of diagnosis, an electrocardioversion was scheduled 2 months later, which was successful. The patient had two more AF episodes lasting longer than 7 days requiring electrocardioversion before surgery, which restored SR in both cases. CB: conduction block; PAF: paroxysmal AF; persAF: persistent AF; AT: activation time.

\subsection{Conduction Disorders in Relation to Spontaneous Termination of AF Episodes}

Thirty-nine patients had spontaneous termination of AF episodes (ST group) and 32 patients had AF episodes that did not terminate spontaneously (NST group).

Patients without spontaneous termination of AF episodes had a prolonged TAT (ST: 134.0 (115.8-153.3) ms vs. NST: 152.5 (136.4-165.1) ms, $p=0.033)$ and AT-BB (ST: 53.5 (39.6-75.8) ms, NST: $72.0(49.6-80.8) \mathrm{ms}$, ST $p=0.009)$. Other conduction characteristics did not differ between the groups. 


\subsection{Conduction Disorders in Relation to P-Wave Duration and a-IAB}

In $48(67.6 \%)$ patients, of whom six patients with persistent AF, an ECG was available for evaluation of P-wave duration and a-IAB. In 27 patients (56.3\%), P-wave duration was $\geq 120 \mathrm{~ms}$ and five patients $(11.1 \%)$ met the criteria of an a-IAB.

Patients with a P-wave duration $\geq 120 \mathrm{~ms}$ had a significantly higher prevalence of $\mathrm{CB}$ and $\mathrm{CCDCB}$ at $\mathrm{BB}(\mathrm{CB}: 8.5 \pm 5.7 \%$ vs. $4.3 \pm 2.5 \%, p=0.001$; $\mathrm{CCDCB}: 10.8 \pm 7.6 \%$ vs. $6.2 \pm 4.2 \%, p=0.012$ ). This was due to a prolongation of the longest $C B$ and $C C D C B$ lines at $\mathrm{BB}$ in these patients (CB: 32(22-40) mm vs. 20 (14-28) mm, $p=0.014$; CCDCB: 52 (30-76) mm vs. $34(20-44) \mathrm{mm}, p=0.026)$. At the PVA, the length of $\mathrm{CCDCB}$ lines was longer in patients with a P-wave duration $\geq 120 \mathrm{~ms}(15.7(12.1-23.8) \mathrm{mm}$ vs. $13.5(10.1-14.9) \mathrm{mm}, p=0.036)$. Furthermore, AT-RA and AT-BB were longer in patients with a P-wave duration $\geq 120 \mathrm{~ms}$ (AT-RA: 89.0 (77.9-104.5) ms vs. 75.0 (64.0-88.0) ms, $p=0.010$; AT-BB: 64.3 (52.3-93.0) ms vs. 50.5 (39.6-56.6) $\mathrm{ms}, p=0.004)$.

Patients with a-IAB also had a higher prevalence of CB at BB (12.7 (6.8-17.8)\% vs. 5.1 $(3.2-8.4) \%, p=0.028)$, which was caused by a prolongation of the longest $C B$ line at $B B$ in these patients ( 40 (30-77) $\mathrm{mm}$ vs. $26(16-34) \mathrm{mm}, p=0.018)$. The maximum $\mathrm{CT}$ at $\mathrm{BB}$ was longer in patients with a-IAB ( 80 (55-99) ms vs. 32 (23.5-27) ms, $p<0.0005)$. Remarkably, the prevalence of $\mathrm{CD}$ at the LA was lower in patients with a-IAB $(0.9(0.3-1.7) \%$ vs. 2.7 $(1.6-3.8) \%, p=0.006)$. At the PVA, the prevalence of CD was higher in patients with a-IAB $(5.8(5.5-6.2) \%$ vs. $3.4(1.8-5.3) \%, p<0.0005)$. Furthermore, patients with a-IAB had a longer AT-RA (117.5 (101.8-175.0) ms vs. $80.3(66.6-93.1) \mathrm{ms}, p<0.0005)$, AT-BB $(92.0(80.5-115.0)$ ms vs. 53.0 (41.0-63.5) $\mathrm{ms}, p=0.002)$, AT-PV (63.0 (51.0-79.8) $\mathrm{ms}$ vs. 41.0 [37.0-53.0 ms, $p=0.005)$ and TAT (213.0 (178.0-240.3) ms vs. $137.0(119.1-152.8) \mathrm{ms}, p<0.0005)$. However, these comparisons are based on only five patients with a-IAB on ECG.

\subsection{Discriminatory Value of Electropathology}

The most optimal ROC-curve for paroxysmal vs. persistent AF patients, patients with vs. without spontaneous termination and patients with vs. without a P-wave duration $\geq$ $120 \mathrm{~ms}$ was of AT-BB (AUC 0.681 [0.544-0.818], TAT (AUC 0.689 [0.562-0.817]) and again AT-BB (AUC 0.749 [0.608-0.890]), respectively. Thus, none of these conduction parameters were clearly discriminatory. We did not construct ROC-curves for patients with vs. without a-IAB, as only five patients met the criteria for a-IAB. The AUC values for all ROC-curves are depicted in the Supplemental Table S3.

\subsection{Correlation of Conduction Parameters with HATCH Score}

The median HATCH score was measured in all paroxysmal AF patients and was 2 [1-3]; only three patients had a score higher than 4 . The HATCH score correlated with CB prevalence at BB (rho 0.326, $p=0.029$ ) and AT-BB (rho 0.378, $p=0.011$ ).

\section{Discussion}

\subsection{Main Findings}

A higher number of conduction disorders was mainly found at $\mathrm{BB}$ in patients with persistent $\mathrm{AF}$, without spontaneous termination of $\mathrm{AF}$, with a P-wave duration $\geq 120 \mathrm{~ms}$ or with a higher HATCH score undergoing cardiac surgery. In patients with persistent $\mathrm{AF}$, there was a higher number of $\mathrm{CCDCB}$ lines and perpendicularly orientated $\mathrm{CB}$ lines. Consequently, both AT-BB and TAT were prolonged.

However, the AUCs of the ROC-curves using electrophysiological parameters to distinguish AF subtypes were only moderately discriminatory.

\subsection{Clinical Classification of $A F$}

Prior studies have demonstrated that persistent AF patients can regress to paroxysmal $\mathrm{AF}$ and even the AF-burden cannot distinguish paroxysmal from persistent $\mathrm{AF}[16,17]$. Shortcomings of the standard clinical classification, P-wave duration and spontaneous termination of AF episodes may be reflected by the absence of differences in conduction 
disorders found in our study. Our study is the first to use multiple clinical classifications to investigate differences in electropathology. However, our measurements were limited to SR. Measurements during programmed electrical stimulation and AF will elucidate direction and frequency dependent conduction disturbances and provide us with additional insights which are necessary for a classification based on severity of electropathology. Additionally, studies incorporating AF progression in relation to electropathology are needed. A classification based on severity of electropathology is requisite to improve on ablation therapies and to select the right patients for the various therapeutic strategies.

\subsection{Conduction Disorders in Relation to AF Classification}

Conduction disorders are generally assumed to play an important role, both in the commencement of AF and its maintenance [18]. Verheule et al. found lower atrial conduction velocities during slow pacing after cardioversion to SR in goats with pacemaker-maintained AF for 6 months compared to 3 weeks. After 6 months, goats had increased endomysial fibrosis, which is the probable explanation for this slowing of conduction during pacing [19]. Various endocardial electrophysiological studies in patients also reported slower conduction at $\mathrm{BB}$ and the $\mathrm{LA}$ in paroxysmal AF patients when compared to persistent $\mathrm{AF}$ patients [6,8-10]. However, BB was never measured directly, as mapping in these studies was performed at the endocardium. Lin et al. found a stepwise increase in SR LA activation time from patients without $\mathrm{AF}$ to paroxysmal $\mathrm{AF}$, persistent $\mathrm{AF}$ and long-standing $\mathrm{AF}$ [9]. We also found a difference in LA activation time; however, this difference disappeared when correcting for possible confounding factors. Our study is the first high-resolution epicardial mapping study to show a difference in TAT and AT at BB during SR between paroxysmal and persistent AF after correction for patient characteristics. This prolongation of AT-BB reflects the advancement of interatrial conduction disorders in patients with persisting AF. As TAT remained significant, even after correction for left atrial enlargement which could also influence TAT, a prolonged AT of BB in patients with persistent AF also resulted in a prolonged TAT. Furthermore, persistent AF was an independent predictor for a longer TAT and $\mathrm{AT}$ at $\mathrm{BB}$ after correcting for $\mathrm{AF}$ prior to onset of the mapping procedure. This correction was made to adjust for the possible interference of electrical remodeling as measurements in some patients were performed shortly after electrocardioversion [20].

\subsection{Bachmann's Bundle in AF}

In an experiment in 1971 by Waldo et al., a perpendicular lesion in BB was found to affect P-wave duration and produce an a-IAB-like P-wave [21]. Consequentially, it was assumed that BB plays a role in AF as a-IAB is associated with AF development and progression [22]. Additionally, Kumagai et al. found, using a sterile pericarditis canine model, that BB seemed to be critical in maintaining AF. During AF, BB was involved in many re-entry circuits and, after creating a full-thickness lesion in $\mathrm{BB}, \mathrm{AF}$ was terminated and sustained AF could not be induced again [23]. At first glance these results may seem to contradict each other, as a full thickness lesion in BB inhibits AF induction while such a lesion also induces a-IAB which is associated with $\mathrm{AF}$ development and progression. However, in the case of a-IAB as seen on the ECG, conduction at BB is probably not blocked in the whole thickness of $\mathrm{BB}$. In our study, more $\mathrm{CCDCB}$ lines and more perpendicularly orientated $C B$ line parts at $B B$ in patients with persistent $A F$ accounted for a prolongation of $\mathrm{SR}$ activation time of $\mathrm{BB}$. These conduction disturbances could lead to a-IAB-like changes on the ECG as conduction finds a faster alternative pathway leading to caudo-cranial activation of the LA. This was the case in our patients, as a P-wave duration of $\geq 120 \mathrm{~ms}$ or a-IAB on ECG correlated with AT-BB.

The pathophysiology behind the association between a-IAB and progression of AF is unclear. The link between persistence of $\mathrm{AF}$ and a longer AT at BB indicating more conduction disturbances can also not be concluded from our data. The higher amount of conduction disturbances indicated by a longer AT in patients with persistent AF found at $\mathrm{BB}$ could merely be the result of a higher susceptibility to AF remodeling of this loca- 
tion. However, the conduction disturbances could also contribute to a more stable AF, as suggested in prior studies [22,23]. AF stability could be the result of the extensive conduction disturbances at BB favoring re-entry in combination with the communicating role of BB linking the RA and LA. A more causal effect of conduction disturbances and the persistence of AF was indicated by the observed differences in conduction disturbances at $\mathrm{BB}$ in patients with a-IAB and/or a longer P-wave duration.

\subsection{HATCH Score and Electropathology}

The HATCH score was developed to predict progression of AF for patients with paroxysmal AF [12]. In follow-up studies, the success of the HATCH score was limited [24,25]. In our study, correlations were found between electrophysiological parameters and HATCH scores. Interestingly, one of these correlations was with AT at BB. However, the resulting correlation coefficients for HATCH scores with conduction parameters indicate only moderate correlations.

\subsection{Limitations}

There may be a bias in our study group as only AF patients in whom SR was restored after electrocardioversion were included. Furthermore, our study population is relatively small and it represents a specific subset of the AF population requiring cardiac surgery, which might not be representative of the general AF population. Our measurements were performed during SR, additional measurements during programmed electrical stimulation and AF will uncover potential direction and frequency-dependent conduction disturbances.

\section{Conclusions}

Using four different AF clinical classification methods, we did not find any conduction parameter during SR differentiating between AF subtypes in patients undergoing cardiac surgery. The observed differences in conduction parameters, mainly found at BB, were only moderately discriminatory. Future studies are needed to establish an objective classification for AF based on quantified features of electropathology in order to provide patient-tailored therapy of AF.

Supplementary Materials: The following are available online at https://www.mdpi.com/article/10 .3390/jcm10132846/s1: Table S1, prevalences of CD, CB and CCDCB per location; Table S2, multivariate analysis; Table S3, ROC curve AUC values.

Author Contributions: Conceptualization, W.F.B.v.d.D. and N.M.S.d.G.; data curation, W.F.B.v.d.D., A.H. and N.M.S.d.G.; formal analysis, W.F.B.v.d.D.; funding acquisition, N.M.S.d.G.; investigation, W.F.B.v.d.D.; methodology, W.F.B.v.d.D., A.H. and L.J.M.E.v.d.D.; project administration, W.F.B.v.d.D.; resources, W.F.B.v.d.D., A.H., L.J.M.E.v.d.D., A.J.J.C.B. and N.M.S.d.G.; supervision, A.J.J.C.B. and N.M.S.d.G.; validation, W.F.B.v.d.D. and N.M.S.d.G.; visualization, W.F.B.v.d.D.; writing-original draft, W.F.B.v.d.D.; writing-review and editing, W.F.B.v.d.D., A.H., L.J.M.E.v.d.D., A.J.J.C.B. and N.M.S.d.G. All authors have read and agreed to the published version of the manuscript.

Funding: N.M.S. de Groot is supported by grants from the Investigator-Initiated Study Program of Biosense Webster, Inc. (ICD 783454), CardioVasculair wetenschappelijk Onderzoek Nederland (CVON) [grantnumber 914728], Nederlandse organisatie voor Wetenschappelijk Onderzoek (NWO) -Vidi [grantnumber 91717339] and Medical Delta.

Institutional Review Board Statement: The study was conducted according to the guidelines of the Declaration of Helsinki, and approved by the Medical Ethical Committee in the Erasmus Medical Center (MEC 2010-054 and MEC 2014-393).

Informed Consent Statement: Informed consent was obtained from all subjects involved in the study.

Data Availability Statement: Data supporting the findings of this study is available on reasonable request from the corresponding author.

Acknowledgments: We would like to thank our colleagues J.A. Bekkers, W.J. van Leeuwen, F.B.S. Oei, F.R.N. van Schaagen, P.C. van de Woestijne, A. Yaksh, C.A. Houck, C.P. Teuwen, C.S. Serban, 
E.A.H. Lanters, E.M.J.P. Mouws, L.N. van Staveren, M.S. van Schie, R. Starreveld, R.K. Kharbanda, M.C. Roos for their contribution to this work and I. Kardys for advice on statistical analysis.

Conflicts of Interest: The authors declare no conflict of interest.

\section{References}

1. Kirchhof, P.; Benussi, S.; Kotecha, D.; Ahlsson, A.; Atar, D.; Casadei, B.; Castella, M.; Diener, H.-C.; Heidbuchel, H.; Hendriks, J.; et al. 2016 ESC Guidelines for the management of atrial fibrillation developed in collaboration with EACTS. Europace 2016, 18, 1609-1678. [CrossRef] [PubMed]

2. Tilz, R.R.; Heeger, C.-H.; Wick, A.; Saguner, A.M.; Metzner, A.; Rillig, A.; Wohlmuth, P.; Reissmann, B.; Lemeš, C.; Maurer, T.; et al. Ten-Year Clinical Outcome After Circumferential Pulmonary Vein Isolation Utilizing the Hamburg Approach in Patients with Symptomatic Drug-Refractory Paroxysmal Atrial Fibrillation. Circ. Arrhythmia Electrophysiol. 2018, 11, e005250. [CrossRef] [PubMed]

3. Dukkipati, S.R.; Neuzil, P.; Kautzner, J.; Petru, J.; Wichterle, D.; Škoda, J.; Čihák, R.; Peichl, P.; Russo, A.D.; Pelargonio, G.; et al. The durability of pulmonary vein isolation using the visually guided laser balloon catheter: Multicenter results of pulmonary vein remapping studies. Heart Rhythm. 2012, 9, 919-925. [CrossRef] [PubMed]

4. Jilek, C.; Ullah, W. Pulmonary vein reconnections or substrate in the left atrium: What is the reason for atrial fibrillation re-currences? A dialogue on a pressing clinical situation. EP Eur. 2019, 21, i12-i20. [CrossRef] [PubMed]

5. Straube, F.; Dorwarth, U.; Hartl, S.; Brueck, B.; Pongratz, J.; Kosmalla, A.; Wankerl, M.; Hoffmann, E. Benefit of ultra-high-density mapping-guided radiofrequency reablation in pulmonary vein isolation non-responders after initial cryoballoon procedure. $E P$ Eur. 2020, 22, 906-915. [CrossRef] [PubMed]

6. O'Donnell, D.A.V.I.D.; Bourke, J.P.; Furniss, S.S. Interatrial transseptal electrical conduction: Comparison of patients with atrial fi-brillation and normal controls. J. Cardiovasc. Electrophysiol. 2002, 13, 1111-1117. [CrossRef] [PubMed]

7. Teh, A.W.; Kistler, P.M.; Lee, G.; Medi, C.; Heck, P.M.; Spence, S.J.; Sparks, P.B.; Morton, J.B.; Kalman, J.M. Electroanatomic Remodeling of the Left Atrium in Paroxysmal and Persistent Atrial Fibrillation Patients Without Structural Heart Disease. J. Cardiovasc. Electrophysiol. 2011, 23, 232-238. [CrossRef]

8. Chang, S.L.; Tai, C.T.; Lin, Y.J.; Wongcharoen, W.; Lo, L.W.; Tuan, T.C.; Udyavar, A.R.; Chang, S.H.; Tsao, H.M.; Hsieh, M.H.; et al. Biatrial Substrate Properties in Patients with Atrial Fibrillation. J. Cardiovasc. Electrophysiol. 2007, 18, 1134-1139. [CrossRef] [PubMed]

9. Lin, Y.; Yang, B.; Garcia, F.C.; Ju, W.; Zhang, F.; Chen, H.; Yu, J.; Li, M.; Gu, K.; Cao, K.; et al. Comparison of left atrial electrophysiologic abnormalities during sinus rhythm in patients with different type of atrial fibrillation. J. Interv. Card. Electrophysiol. 2014, 39, 57-67. [CrossRef] [PubMed]

10. Sonoda, K.; Okumura, Y.; Watanabe, I.; Nagashima, K.; Kofune, M.; Mano, H.; Kogawa, R.; Sasaki, N.; Ohkubo, K.; Nakai, T.; et al. Three-Dimensional High-Density Bipolar Contact Mapping of Left Atrial Endocardial Activation During Sinus Rhythm in Patients with Atrial Fibrillation. Int. Heart J. 2013, 54, 285-288. [CrossRef]

11. Mouws, E.M.; Van Der Does, L.J.M.E.; Kik, C.; Lanters, E.A.H.; Teuwen, C.P.; Knops, P.; Bogers, A.J.; De Groot, N.M. Impact of the arrhythmogenic potential of long lines of conduction slowing at the pulmonary vein area. Heart Rhythm. 2019, 16, 511-519. [CrossRef] [PubMed]

12. de Vos, C.B.; Pisters, R.; Nieuwlaat, R.; Prins, M.H.; Tieleman, R.G.; Coelen, R.; van den Heijkant, A.C.; Allessie, M.A.; Crijns, H.J. Progression from Paroxysmal to Persistent Atrial Fibrillation: Clinical Correlates and Prognosis. J. Am. Coll. Cardiol. 2010, 55, 725-731. [CrossRef]

13. de Luna, A.B.; Baranchuk, A.; Robledo, L.A.E.; van Roessel, A.M.; Martinez-Selles, M. Diagnosis of in-teratrial block. J. Geriatr. Cardiol. 2017, 14, 161-165.

14. Van Der Does, L.J.M.E.; Yaksh, A.; Kik, C.; Knops, P.; Lanters, E.A.H.; Teuwen, C.P.; Oei, F.B.S.; Van De Woestijne, P.C.; Bekkers, J.A.; Bogers, A.J.J.C.; et al. QUest for the Arrhythmogenic Substrate of Atrial fibRillation in Patients Undergoing Cardiac Surgery (QUASAR Study): Rationale and Design. J. Cardiovasc. Transl. Res. 2016, 9, 194-201. [CrossRef] [PubMed]

15. Lanters, E.A.; Yaksh, A.; Teuwen, C.P.; van der Does, L.J.; Kik, C.; Knops, P.; van Marion, D.M.S.; Brundel, B.; Bogers, A.; Allessie, M.A.; et al. Spatial distribution of conduction disorders during sinus rhythm. Int. J. Cardiol. 2017, 249, 220-225. [CrossRef] [PubMed]

16. Charitos, E.I.; Purerfellner, H.; Glotzer, T.V.; Ziegler, P.D. Clinical classifications of atrial fibrillation poorly reflect its temporal persistence: Insights from 1,195 patients continuously monitored with implantable devices. J. Am. Coll. Cardiol. 2014, 63, 2840-2848. [CrossRef] [PubMed]

17. Sugihara, C.; Veasey, R.; Freemantle, N.; Podd, S.; Furniss, S.; Sulke, N. The development of AF over time in patients with permanent pacemakers: Objective assessment with pacemaker diagnostics demonstrates distinct patterns of AF. EP Eur. 2015, 17, 864-870. [CrossRef] [PubMed]

18. Kumar, S.; Teh, A.W.; Medi, C.; Kistler, P.M.; Morton, J.B.; Kalman, J.M. Atrial remodeling in varying clinical substrates within beating human hearts: Relevance to atrial fibrillation. Prog. Biophys. Mol. Biol. 2012, 110, 278-294. [CrossRef] [PubMed]

19. Verheule, S.; Tuyls, E.; Van Hunnik, A.; Kuiper, M.; Schotten, U.; Allessie, M. Fibrillatory Conduction in the Atrial Free Walls of Goats in Persistent and Permanent Atrial Fibrillation. Circ. Arrhythmia Electrophysiol. 2010, 3, 590-599. [CrossRef] [PubMed] 
20. Duytschaever, M.; Danse, P.; Allessie, M. Supervulnerable phase immediately after termination of atrial fibrillation. J. Cardiovasc. Electrophysiol. 2002, 13, 267-275. [CrossRef] [PubMed]

21. Waldo, A.L.; Bush, H.L., Jr.; Gelband, H.; Zorn, G.L., Jr.; Vitikainen, K.J.; Hoffman, B.F. Effects on the canine p wave of discrete lesions in the specialized atrial tracts. Circ. Res. 1971, 29, 452-467. [CrossRef] [PubMed]

22. Tse, G.; Wong, C.W.; Gong, M.; Wong, W.T.; Bazoukis, G.; Wong, S.H.; Li, G.; Wu, W.K.K.; Tse, L.A.; Lampropoulos, K.; et al. Predictive value of inter-atrial block for new onset or recurrent atrial fibrillation: A systematic review and meta-analysis. Int. J. Cardiol. 2018, 250, 152-156. [CrossRef] [PubMed]

23. Kumagai, K.; Uno, K.; Khrestian, C.; Waldo, A.L. Single site radiofrequency catheter ablation of atrial fibrillation: Studies guided by simultaneous multisite mapping in the canine sterile pericarditis model. J. Am. Coll. Cardiol. 2000, 36, 917-923. [CrossRef]

24. Barrett, T.W.; Self, W.H.; Wasserman, B.S.; McNaughton, C.D.; Darbar, D. Evaluating the hatch score for predicting progression to sustained atrial fibrillation in ed patients with new atrial fibrillation. Am. J. Emerg. Med. 2013, 31, 792-797. [CrossRef]

25. Potpara, T.S.; Stankovic, G.R.; Beleslin, B.D.; Polovina, M.M.; Marinkovic, J.M.; Ostojic, M.C.; Lip, G.Y. A 12-year follow-up study of patients with newly diagnosed lone atrial fibrillation: Implications of arrhythmia progression on prognosis: The belgrade atrial fibrillation study. Chest 2012, 141, 339-347. [CrossRef] [PubMed] 